\title{
LOS EFECTOS DE LOS PROGRAMAS SOCIALES EN LA SALUD DE LA POBLACIÓN EN CONDICIÓN DE POBREZA: EVIDENCIAS A PARTIR DE LAS EVALUACIONES DE IMPACTO DEL PRESUPUESTO POR RESULTADOS A PROGRAMAS SOCIALES EN PERÚ
}

\author{
Denice Cavero-Arguedas ${ }^{1, a, b}$, Viviana Cruzado de la Vega ${ }^{1,2, a, c}$, Gabriela Cuadra-Carrasco ${ }^{1,2, a, d}$
}

\begin{abstract}
RESUMEN
Este artículo describe la experiencia en la gestión de evaluaciones de impacto del Ministerio de Economía y Finanzas (MEF) como uno de los instrumentos del presupuesto por resultados, y documenta el diseño de las evaluaciones de impacto a tres programas sociales emblemáticos y los resultados obtenidos, haciendo énfasis en aquellos vinculados a la salud de su población objetivo. Estos son el Servicio de Acompañamiento a Familias (SAF) del Programa Nacional Cuna Más, el Programa Nacional de Apoyo Directo a los Más Pobres JUNTOS y el Programa Nacional de Asistencia Solidaria Pensión 65. Los resultados muestran que el SAF generó mejoras en el desarrollo cognitivo y de lenguaje en los niños mas no en las prácticas de cuidado infantil de las madres, ni en el estado nutricional de los niños. JUNTOS logró incrementos en el gasto per cápita, gasto en alimentos, disminución de la severidad de pobreza y mejoras en el logro educativo. Sin embargo, no se encontraron resultados significativos en la mayoría de indicadores de salud prenatal ni estado nutricional infantil. Pensión 65 mejoró la salud emocional del adulto mayor (depresión, autovaloración), pero no se evidenció aumentos en el uso de servicios de salud por parte de los adultos mayores ni mejoras en su salud física. Se recomienda que dichos programas fortalezcan sus diseños y acciones de articulación con el Ministerio de Salud y entre diferentes niveles de Gobierno, cuando corresponda, a fin de mejorar la oferta y contenidos de las prácticas saludables y optimizar la prestación de los servicios de salud.
\end{abstract}

Palabras clave: Presupuesto por resultados; Evaluación de programas; Transferencias condicionadas; Desarrollo infantil (Fuente: DeCS, BIREME)

\section{EFFECTS OF SOCIAL PROGRAMS ON INDIGENT POPULATION HEALTH: EVIDENCE FROM RESULTS-BASED BUDGETING'S IMPACT EVALUATIONS TO SOCIAL PROGRAMS IN PERU}

\begin{abstract}
This article describes the experience of the MEF's impact evaluation management as one of the RBB instruments and documents the design and results obtained from three impact evaluations of the most emblematic government social programs. The Service of Visiting Families (SAF) of the National Program "Cuna Mas", conditional cash transfer Program "JUNTOS" and National Program "Pension 65" focusing on objective population's health the outcomes. Among the main results, it was found the SAF generated improvements in cognitive and communication development in children, but had no impact on mothers' child care practices or children's nutritional status. In the case of JUNTOS, there were increases in per capita spending, food expenditure, decreases in severity and poverty gap, increases in school attendance and reductions of school dropout. However, no significant results were found in most indicators of prenatal health, child health, or chronic malnutrition. In the case of Pension 65, there were increases in household consumption and improvements in elderly's emotional health (depression, self valoration); but there was no evidence of increases in the use of health services by the elderly or improvements in their physical health. Therefore, it is recommended that such programs boost their designs and inter-sectoral coordination with MINSA and subnational institutions, in order to improve contents of healthy practices and child care, and optimize the provision of health and education services, in order to meet the demands of their users.
\end{abstract}

Key words: Results based budgeting; Program evaluation; Conditional cash transfer programs; Child development (Source: MeSH, NLM)

\footnotetext{
Ministerio de Economía y Finanzas. Lima, Perú.

Pontificia Universidad Católica del Perú. Lima, Perú.

Economista; ${ }^{b}$ Máster en Economía del Desarrollo; ${ }^{\mathrm{c}}$ Magíster en Economía Aplicada y Magíster en Estadística ${ }^{\mathrm{e}}$ Investigación Operativa; ${ }^{\mathrm{d}}$ Máster en Economía y Máster en Economía y Finanzas.

Recibido: 15/08/2017 Aprobado: 20/09/2017 En línea: 29/09/2017
} 


\section{INTRODUCCIÓN}

A mediados de los noventa, los países latinoamericanos implementaron importantes cambios en sus políticas sociales tales como el incremento del gasto social, creación de instituciones rectoras de la política social e introducción de programas que buscaban atender problemas específicos de la población pobre. Así, surgieron programas de cuidado y desarrollo infantil enfocados a mejorar la nutrición, el desarrollo cognitivo y psicosocial de los niños; programas de transferencias monetarias condicionadas orientados a aliviar la transmisión intergeneracional de la pobreza; programas de pensiones no contributivas destinados a brindar protección social a los adultos mayores, entre otros.

Paralelamente, nació el interés por evaluar los efectos de estas intervenciones a partir de evaluaciones experimentales y cuasiexperimentales. Se han encontrado efectos positivos y significativos en desarrollo cognitivo y psicosocial de los niños ${ }^{(1-3)}$. No obstante, los efectos sobre la salud de los mismos no son concluyentes. Algunos han encontrado que la mayor exposición ha contribuido al cumplimiento de esquemas de vacunación; mientras que otros han hallado efectos negativos en dichas variables $\mathrm{y}$ escasos efectos sobre la desnutrición crónica infantil (2-3).

Los estudios efectuados sobre programas de transferencias monetarias han hallado efectos positivos en el cumplimiento de las condicionalidades vinculadas con la asistencia a los centros de salud y a la escuela ${ }^{(4)} e$ incrementos en el consumo per cápita del hogar y el gasto en alimentos ${ }^{(5-7)} y$, en algunos casos, han logrado reducir la pobreza y su severidad ${ }^{(5,8)}$. Sin embargo, los efectos sobre el estado nutricional y logros cognitivos de los niños no son consistentes ${ }^{(9-12)}$.

Las evaluaciones a programas de pensiones no contributivas llevadas a cabo en Argentina, Brasil y México, han encontrado la disminución de la oferta laboral de los adultos mayores e incrementos del consumo de los hogares ${ }^{(13-16)}$. Además, aquellos que han evaluado la salud del adulto mayor han hallado efectos positivos en la salud mental, pero no en la salud física ${ }^{(16)}$.

En el Perú, el 2005 se implementó el Programa Juntos en 2005 y en octubre de 2011 se creó el Ministerio de Desarrollo e Inclusión Social (MIDIS) con el objeto de liderar la agenda de inclusión social, a partir de la estrategia nacional "Incluir para crecer". Dicha esta estrategia se encuentra basada en un enfoque del ciclo de vida y es implementada a través de diferentes programas que atienden a la población en condición de pobreza y exclusión en distintos rangos de edad (17).
La estrategia abarca los siguientes ejes: (i) nutrición infantil, dirigido a niños de 0-3 años y madre gestante; (ii) desarrollo infantil temprano, dirigido a niños de 0-5 años; (iii) desarrollo integral de la niñez y adolescencia, dirigido a la población de 6-17 años; (iv) inclusión económica, dirigido a personas en edad productiva, entre 18 y 64 años; y (v) protección al adulto mayor, concentrado en la población mayor a 65 años. El Programa Nacional Cuna Más (PNCM) se incluye en el segundo eje dos, el Programa de Apoyo a los más Pobres Juntos se enmarca dentro de los ejes i a iii, y el Programa Nacional de Asistencia Solidaria Pensión 65, en el eje $v$.

Dichos programas, además, han contado con evaluaciones de impacto lideradas desde el sector público a través del Ministerio de Economía y Finanzas (MEF) como parte de los esfuerzos de generar evidencia para la toma de decisiones en el marco del Presupuesto por Resultados $(\mathrm{PpR})$. El presente artículo documenta dichas experiencias y reporta los efectos de sus evaluaciones de impacto sobre sus principales resultados y sobre indicadores de salud en su población objetivo.

Para ello, en la siguiente sección se presenta la experiencia en la gestión de evaluaciones de impacto del MEF como uno de los instrumentos del PpR. En la tercera sección se describe el diseño de evaluación y resultados de los programas anteriormente señalados. En la cuarta sección se presenta el balance y las conclusiones.

\section{EVALUACIONES DE IMPACTO COMO INSTRUMENTO DEL PRESUPUESTO POR RESULTADOS}

A fin de contribuir a una mejora en la eficiencia y eficacia del gasto público, el MEF ha venido impulsando, desde 2008, el proceso de reforma del PpR. Este busca fortalecer la relación entre el presupuesto y los resultados mediante el uso sistemático de la información de desempeño que sustenten las decisiones de asignación presupuestal.

Uno de los instrumentos del PpR que contribuye a generar información de desempeño son las evaluaciones independientes. Estas incluyen a las evaluaciones de diseño y ejecución presupuestal, y a las evaluaciones de impacto. Las primeras constituyen un instrumento de evaluación del diseño y de la implementación operativa de las intervenciones públicas. Las segundas estiman el efecto atribuible a dichas intervenciones sobre el logro de un conjunto de resultados esperados.

La experiencia en la gestión y realización de evaluaciones de impacto como un instrumento del PpR se inicia en 
2010. Las primeras evaluaciones de impacto que se realizaron desde la Dirección de Calidad de Gasto Público (DCGP) tuvieron un enfoque retrospectivo a partir del uso de metodologías cuasiexperimentales que emplearon las encuestas de hogares que realiza el Instituto Nacional de Estadística e Informática (INEI) y registros administrativos para la estimación de sus impactos.

En 2012 se destinaron cantidades significativas de recursos presupuestales para la creación de nuevos programas sociales prioritarios para el gobierno, además, se empezó a planificar un conjunto de evaluaciones priorizando un enfoque prospectivo, es decir, donde el diseño y la implementación de las evaluaciones se realiza conjuntamente. Esto permitió utilizar diseños metodológicos más rigurosos y definir ex ante a los grupos de tratamiento y control, con muestras representativas y operativos de campo debidamente planificados.

Bajo esta modalidad, se emprendieron las evaluaciones de impacto al Servicio de Acompañamiento a Familias (SAF) del PNCM a partir de un diseño experimental, la evaluación de impacto a Pensión 65 a partir de un diseño de regresión discontinua y la evaluación de impacto a Juntos, que empleó una metodología de diferencias en diferencias a partir de una muestra panel de hogares. En los dos primeros, las mediciones basales y de seguimiento se realizaron en 2012 y 2015 . En el caso de Juntos, dichas mediciones se hicieron en 2011 y 2016.

Para la conducción de dichas evaluaciones se estableció un equipo técnico, conformado por representantes de la DCGP del MEF, de la Dirección General de Seguimiento y Evaluación del MIDIS y del programa evaluado. En algunos casos se contó con la asistencia técnica de organismos multilaterales como el Banco Interamericano de Desarrollo (BID), y agencias de investigación especializadas como Innovations for Poverty Action (IPA) y MacroConsult. Los operativos de campo para el levantamiento de datos fueron realizados por el INEI a solicitud del MEF.

\section{RESULTADOS DE LAS EVALUACIONES DE IMPACTO DEL PPR}

\section{CASO 1. EVALUACIÓN DE IMPACTO AL SERVICIO DE ACOMPAÑAMIENTO A FAMILIAS DEL PNCM ${ }^{(18,19)}$}

El SAF busca mejorar el nivel de desarrollo en la dimensión motora, lenguaje, cognitiva y socioemocional de niños menores de 3 años que viven en situación de pobreza en zonas rurales y con elevadas tasas de desnutrición. Para ello, ofrece visitas semanales al hogar del niño, en las cuales, facilitadoras de la comunidad capacitadas por el programa trabajan con la madre y el niño en una serie de actividades que buscan mejorar sus prácticas de crianza y la calidad de las interacciones con los niños a través de actividades de juego que promueven el desarrollo infantil. Las familias usuarias también reciben materiales de promoción de prácticas de salud y nutrición.

El objetivo principal de la evaluación fue medir los impactos del SAF en el nivel del desarrollo de los niños menores de 36 meses. No obstante, dado que la acción directa del SAF se da través de la madre, se analizaron también los cambios en las prácticas de cuidado y crianza de la madre (Tabla 2). Adicionalmente, se evaluó el impacto del SAF sobre otros indicadores como el estatus nutricional de los niños.

El equipo de trabajo de la evaluación planteó un diseño de evaluación experimental, aprovechando la implementación gradual de la intervención para atender a su población objetivo, para lo cual se aleatorizó el orden de entrada de distritos potencialmente beneficiarios. El marco muestral estuvo constituido por 531 distritos focalizados. Para la selección de la muestra, los distritos se ordenaron según su nivel de pobreza y se eligieron aleatoriamente 60 tríos. En cada trío, se asignaron aleatoriamente dos distritos al grupo de tratamiento y uno al grupo de control. Los primeros se incorporarían al SAF desde 2013, y los segundos, inmediatamente después del levantamiento de la línea de seguimiento. La muestra estuvo conformada por 5375 niños distribuidos en 360 centros poblados de 180 distritos en 12 departamentos.

Para medir desarrollo infantil temprano se aplicaron dos pruebas. A la muestra conformada por 5339 niños distribuidos en 360 centros poblados de 180 distritos en 12 departamentos se le aplicó el cuestionario de edades y etapas, Ages and Stages Questionnaire ASQ-3 (20), que es una prueba de tamizaje para niños de entre 1 y 66 meses. A la submuestra de 1038 niños menores de 42 meses se les aplicó la escala de Bayley Scales of Infant and Toddler Development - III (20). En el primer caso, se encontró que el $64 \%$ de niños asignados al grupo de tratamiento recibió por lo menos una visita mientras que en el segundo caso, este porcentaje fue de $83 \%$.

El diseño experimental buscó asegurar grupos equivalentes en la mayoría de variables (Tabla 1). Se midieron impactos del programa a través de dos estimadores: el de intención de tratamiento (intent-to-treat o ITT), que considera a todo el grupo de tratamiento, y el de tratamiento en los efectivamente tratados (treatment-on-the-treated o TOT), que considera solo al grupo que recibió, por lo menos, una visita. Los estimadores ITT reportaron impactos modestos en las áreas de resolución de problemas y de comunicación. El estimador TOT reportó efectos de mayor magnitud. En las áreas de motricidad fina y socioemocional, los impactos fueron ligeramente menores (Tabla 2). 
Tabla 1. Características de la muestra en la línea de base de las intervenciones evaluadas

\begin{tabular}{|c|c|c|c|c|c|}
\hline & \multirow{2}{*}{$\begin{array}{l}\text { Unidad de } \\
\text { Medida }\end{array}$} & \multirow{2}{*}{$\mathbf{N}$} & \multicolumn{2}{|c|}{ Promedio } & \multirow{2}{*}{$\begin{array}{c}\text { Valor } \\
\mathbf{p}^{\wedge}\end{array}$} \\
\hline & & & Tratados & Controles & \\
\hline \multicolumn{6}{|l|}{ CUNA MÁS } \\
\hline Edad del niño (meses) & meses & 5339 & 12,83 & 12,70 & $>0,10$ \\
\hline Sexo del niño (1=mujeres) & proporción & 5339 & 0,49 & 0,49 & $>0,10$ \\
\hline Desnutrición Crónica del niño & tasa & 5186 & 0,37 & 0,38 & $>0,10$ \\
\hline Desnutrición global del niño & tasa & 5190 & 0,08 & 0,08 & $>0,10$ \\
\hline \multicolumn{6}{|l|}{ Características del cuidador del hogar } \\
\hline Años de educación (madre) & años & 5065 & 6,71 & 6,29 & $<0,05$ \\
\hline Proporción de indígenas (madre) & proporción & 5339 & 0,08 & 0,16 & $<0,05$ \\
\hline Proporción con televisor & proporción & 5339 & 0,52 & 0,45 & $<0,05$ \\
\hline Proporción con refrigeradora & proporción & 5339 & 0,80 & 0,07 & $>0,10$ \\
\hline Proporción con cocina a gas & proporción & 5339 & 0,43 & 0,37 & $<0,10$ \\
\hline Proporción con celular & proporción & 5339 & 0,61 & 0,59 & $>0,10$ \\
\hline Proporción con piso de tierra & proporción & 5339 & 0,26 & 0,26 & $>0,10$ \\
\hline Proporción con electricidad & proporción & 5339 & 0,77 & 0,74 & $>0,10$ \\
\hline Proporción con agua (dentro de la vivienda) & proporción & 5339 & 0,59 & 0,58 & $>0,10$ \\
\hline Proporción con servicio higiénico (dentro de la vivienda) & proporción & 5339 & 0,27 & 0,20 & $<0,05$ \\
\hline \multicolumn{6}{|l|}{ JUNTOS } \\
\hline Gasto per cápita del hogar & S/ promedio & 1978 & 162,36 & 163,72 & $>0,10$ \\
\hline Gasto per cápita básico del hogar & S/ promedio & 1978 & 115,86 & 115,06 & $>0,10$ \\
\hline Hogar es pobre & proporción & 1978 & 0,93 & 0,92 & $>0,10$ \\
\hline Hogar es pobre extremo & proporción & 1978 & 0,69 & 0,66 & $>0,10$ \\
\hline Edad del jefe del hogar & años & 1978 & 38,12 & 41,05 & $<0,01$ \\
\hline Sexo del jefe del hogar (1=varón) & proporción & 1978 & 0,83 & 1,17 & $>0,10$ \\
\hline Años de educación del jefe del hogar & años & 1978 & 1,83 & 1,67 & $>0,10$ \\
\hline Vivienda propia & proporción & 1978 & 0,67 & 0,70 & $>0,10$ \\
\hline Pared de ladrillo & proporción & 1978 & 0,02 & 0,05 & $>0,10$ \\
\hline Techo de concreto o teja & proporción & 1978 & 0,35 & 0,28 & $>0,10$ \\
\hline Pisos de parquet, loza, asfalto, madera o cemento & proporción & 1978 & 0,07 & 0,14 & $<0,10$ \\
\hline Hogar tiene acceso a agua de red & proporción & 1978 & 0,43 & 0,36 & $>0,10$ \\
\hline Hogar tiene acceso a desagüe de red pública & proporción & 1978 & 0,10 & 0,06 & $<0,10$ \\
\hline Hogar tiene acceso a electricidad de red pública & proporción & 1978 & 0,68 & 0,81 & $<0,01$ \\
\hline Cocina con leña & proporción & 1978 & 0,82 & 0,77 & $>0,10$ \\
\hline Número de miembros del hogar & número & 1978 & 4,48 & 4,49 & $>0,10$ \\
\hline Número de miembros del hogar menores de 19 años & proporción & 1978 & 2,60 & 2,52 & $>0,10$ \\
\hline Altitud del CCPP (m s.n.m.) & número & 1978 & 2560,88 & 2462,07 & $>0,10$ \\
\hline Costa & proporción & 1978 & 0,08 & 0,12 & $>0,10$ \\
\hline Sierra & proporción & 1978 & 0,74 & 0,71 & $>0,10$ \\
\hline Selva & proporción & 1978 & 0,18 & 0,18 & $>0,10$ \\
\hline \multicolumn{6}{|l|}{ PENSIÓN $65^{\wedge \wedge}$} \\
\hline Edad del adulto mayor & años & 3342 & 71,21 & 71,00 & $>0,10$ \\
\hline Sexo del adulto mayor (masculino $=1$ ) & proporción & 3342 & 0,52 & 0,50 & $>0,10$ \\
\hline Casado & proporción & 3342 & 0,75 & 0,70 & $>0,10$ \\
\hline Años de escolaridad & años & 3342 & 4,85 & 4,46 & $>0,10$ \\
\hline Trabajó la semana previa por un salario & proporción & 3342 & 0,54 & 0,58 & $>0,10$ \\
\hline Horas que trabajó la semana previa por un salario & horas & 3342 & 15,91 & 17,42 & $>0,10$ \\
\hline Tiene hipertensión & proporción & 3342 & 0,32 & 0,32 & $>0,10$ \\
\hline Circunferencia de cintura & centímetros & 3342 & 88,75 & 88,06 & $>0,10$ \\
\hline IMC & $\mathrm{kg} / \mathrm{m}^{2}$ & 3342 & 23,59 & 23,54 & $>0,10$ \\
\hline Escala de memoria & índice & 3342 & 11,49 & 11,61 & $>0,10$ \\
\hline Satisfacción (escala del 0 a 1) & índice & 3342 & 0,73 & 0,75 & $>0,10$ \\
\hline Empoderamiento (escala del 0 a 1 ) & índice & 3342 & 0,88 & 0,89 & $>0,10$ \\
\hline Contribución (escala del 0 a 1) & índice & 3342 & 0,80 & 0,81 & $>0,10$ \\
\hline Autovaloración (escala del 0 a 1) & índice & 3342 & 0,60 & 0,60 & $>0,10$ \\
\hline
\end{tabular}

*** Valor $p<0,01,{ }^{* *}$ Valor $p<0,05,{ }^{*}$ Valor $p<0,1$;

$\wedge \mathrm{Ho}:-=0$

$\wedge \wedge$ test de medias condicionado

Fuente: elaboración propia a partir de los informes de evaluación 
Tabla 2. Impactos estimados de Cuna Más

\begin{tabular}{|c|c|c|c|c|c|}
\hline \multirow[b]{2}{*}{ Indicadores de resultado } & \multirow[b]{2}{*}{$\mathbf{N}$} & \multicolumn{2}{|c|}{ Intention-to-treat (ITT) } & \multicolumn{2}{|c|}{ Treatment on treated (TOT) } \\
\hline & & $\beta$ & Error estándar & $\beta$ & Error estándar \\
\hline \multicolumn{6}{|l|}{ Cuestionario de edad y etapas } \\
\hline Resolución de problemas & 5339 & $0,06^{* *}$ & 0,03 & $0,098^{* *}$ & 0,05 \\
\hline Comunicación & 5339 & $0,08^{* *}$ & 0,03 & $0,122^{\star *}$ & 0,05 \\
\hline Motricidad fina & 5339 & $0,06^{*}$ & 0,04 & $0,094^{*}$ & 0,05 \\
\hline Personal-social & 5339 & $0,07^{*}$ & 0,04 & $0,102^{*}$ & 0,06 \\
\hline Motricidad gruesa & 5339 & $-0,01$ & 0,03 & $-0,012$ & 0,05 \\
\hline Total (todas las áreas) & 5339 & $0,06^{*}$ & 0,03 & $0,098^{* *}$ & 0,04 \\
\hline \multicolumn{6}{|l|}{ Bayley-III: } \\
\hline Desarrollo cognitivo & 1038 & $0,25^{\star \star \star}$ & 0,08 & $0,295^{\star * *}$ & 0,09 \\
\hline Leguaje receptivo & 1038 & $0,16^{* *}$ & 0,06 & $0,193^{* * *}$ & 0,079 \\
\hline Lenguaje expresivo & 1038 & $-0,11$ & 0,07 & $-0,132$ & 0,08 \\
\hline Motricidad fina & 1038 & 0,03 & 0,07 & 0,04 & 0,08 \\
\hline Total (todas las áreas) & 1038 & $0,14^{*}$ & 0,07 & $0,165^{*}$ & 0,09 \\
\hline \multicolumn{6}{|l|}{ Indicadores de cuidado familiar } \\
\hline Actividades de juego (FA, puntaje Z) & 5339 & $0,20^{\star * *}$ & 0,03 & & \\
\hline Juguetes caseros & 5268 & $0,08^{* * *}$ & 0,02 & & \\
\hline Juguetes comprados & 5268 & $0,02^{*}$ & 0,01 & & \\
\hline Juguetes con objetos del hogar & 5268 & $-0,01$ & 0,03 & & \\
\hline Libros para adulto & 5268 & $-0,04^{* *}$ & 0,02 & & \\
\hline Periódicos & 5268 & $-0,03^{* *}$ & 0,01 & & \\
\hline Materiales de juegos (FA, puntaje Z) & 5268 & 0,05 & 0,04 & & \\
\hline \multicolumn{6}{|l|}{ Disciplina y formas de felicitar al niño } \\
\hline Gritos e insultos & 5339 & $-0,04^{* * *}$ & 0,01 & & \\
\hline Golpes con correa (o algún otro objeto) & 5339 & $-0,02^{* *}$ & 0,01 & & \\
\hline Felicitar verbalmente o aplaudir & 5339 & $0,08^{* * *}$ & 0,01 & & \\
\hline Dar una recompensa & 5339 & $-0,03^{* * *}$ & 0,01 & & \\
\hline Abrazar, consentir, ser cariñoso & 5339 & $-0,02$ & 0,01 & & \\
\hline Reírse juntos & 5339 & 0,00 & 0,01 & & \\
\hline \multicolumn{6}{|l|}{ Calidad del hogar } \\
\hline Hogar duro (FA, puntaje Z) & 5339 & 0,04 & 0,03 & & \\
\hline Hogar frio (FA, puntaje Z) & 5339 & $-0,02$ & 0,05 & & \\
\hline \multicolumn{6}{|l|}{ Conocimiento sobre desarrollo infantil } \\
\hline Puntaje KIDI (FA, puntaje Z) & 5211 & 0,00 & 0,04 & & \\
\hline Puntaje Cuna Más (FA, puntaje Z) & 5211 & $-0,05$ & 0,05 & & \\
\hline \multicolumn{6}{|l|}{ Educación Inicial } \\
\hline Participación en educación inicial & 3641 & $0,09^{* * *}$ & 0,02 & & \\
\hline \multicolumn{6}{|l|}{ Nutrición } \\
\hline Peso por edad (puntaje Z) & 5328 & 0,03 & 0,02 & & \\
\hline Peso por talla (puntaje Z) & 5323 & 0,02 & 0,02 & & \\
\hline IMC por talla (puntaje Z) & 5321 & 0,00 & 0,02 & & \\
\hline Talla por edad (puntaje Z) & 5329 & $0,04^{*}$ & 0,02 & & \\
\hline Desnutrición global (peso por edad) & 5328 & $-0,01^{* *}$ & 0,01 & & \\
\hline Desnutrición crónica (talla por edad) & 5329 & $-0,01$ & 0,01 & & \\
\hline Desnutrición aguda (peso por talla) & 5323 & 0,00 & 0,00 & & \\
\hline
\end{tabular}

${ }^{* * *}$ Valor $p<0,01,{ }^{* *}$ Valor $p<0,05,{ }^{*}$ Valor $p<0,1$;

Ho: $=0$

${ }^{\wedge}$ Metodología: aleatorización 
El estimador ITT sobre la submuestra a la que se aplicó el instrumento de medición Bayley, donde la cobertura de afiliación y el número de visitas fue más alta que la muestra total, reportó efectos de casi el doble en desarrollo cognitivo y lenguaje; mientras que los estimadores del TOT fueron aún mayores, con impactos moderados en dichas dimensiones (Tabla 2).Se identificó como posibles mediadores de los impactos del SAF una mayor frecuencia de actividades de juego, mayor disponibilidad de juguetes caseros y menos prácticas violentas de disciplina.

Adicionalmente, se exploró si el programa tuvo algún impacto en el estatus nutricional del niño. Ello se hizo a través de siete indicadores: peso para la edad, talla para la edad, el peso para la talla, índice de masa corporal y las prevalencias de la desnutrición global, crónica y aguda. Solo se hallaron resultados con significancia estadísticas para dos de los siete indicadores. En particular, los estimadores del ITT reportaron efectos modestos equivalentes a una reducción de 1,2 puntos porcentuales en la desnutrición global sobre una media de $7,9 \%$ del grupo de control y un incremento de 3,6 desviaciones estándar en el puntaje $Z$ score de talla para la edad (Tabla 2).

\section{CASO 2. EVALUACIÓN DE IMPACTO AL PROGRAMA NACIONAL DE APOYO DIRECTO A LOS MÁS POBRES (JUNTOS) (21)}

La misión de JUNTOS es contribuir a la reducción de la pobreza y su transmisión intergeneracional mediante la entrega bimensual de un incentivo monetario de S/ 200 a los hogares pobres. El incentivo busca promover el acceso a los servicios de salud, nutrición y educación, y busca, mejorar la capacidad de consumo de las familias usuarias.

La evaluación tuvo como objetivo medir los impactos de Juntos a través de los siguientes canales: i) cumplimiento de corresponsabilidades vinculadas con la salud materno-infantil y educación; ii) asignación del subsidio, y iii) conocimientos adquiridos por los padres a partir de la información brindada por el programa. La interacción de dichos mecanismos generaría un mayor nivel de capital humano.

Los datos empleados provinieron de dos encuestas ad hoc, aplicadas en 2011 y en 2016 a un panel de 1978 hogares, de los cuales 1237 fueron intervenidos y 741 fueron controles. Ambos grupos guardan bastante similitud en casi todas las características relevantes en sus mediciones basales (Tabla 1).

Para obtener el estimador de diferencias en diferencias, se compararon los resultados de los hogares/individuos tratados y los hogares/individuos control, antes y después de la intervención. Además, se aplicaron pruebas placebo para verificar el cumplimiento de la hipótesis de que, las trayectorias del grupo de control replicarían las trayectorias que enfrentaría el grupo de tratamiento en ausencia de la intervención.

Los resultados obtenidos evidencian que JUNTOS generó un incremento en el gasto per cápita de los hogares, gasto en alimentos y gasto básico. Ello permitió que la severidad de la pobreza y la brecha, así como la severidad de la pobreza extrema disminuyesen (resultados similares a los encontrados por otros autores) ${ }^{(5)}$. Sin embargo, el programa no tuvo efectos sobre la tasa de pobreza ni sobre la tasa de pobreza extrema (Tabla 3).

En materia de salud, nutrición y prácticas saludables el programa evaluó 22 indicadores, sin embargo, no se hallaron efectos en la mayoría de ellos. En la cadena causal relacionada con la salud prenatal, no hubo efectos en el número de controles prenatales, edad del primer control, ni si realizaron su primer control en el primer trimestre. Por tanto, tampoco en parto institucional, incremento de la talla al nacer, o peso al nacer. En el primer caso la ausencia de efectos podría deberse a los mayores costos de desplazamiento de las gestantes o a que las coberturas podrían ser altas. En la cadena causal vinculada con la salud infantil, a diferencia de otros autores ${ }^{(22-24)}$, se hallaron efectos significativos en el cumplimiento de las pautas de los controles más no en el número de controles por grupo etario, y efectos no robustos en el cumplimiento del esquema de vacunación. Tampoco se encontraron impactos en la desnutrición crónica infantil.

Sin embargo, el programa tuvo efectos significativos en la mayor parte de los indicadores educativos: aumentó la asistencia escolar y redujo la deserción escolar. Además, incrementó el tiempo destinado a actividades educativas (Tabla 3). No obstante, al desagregar los resultados por sexo y por grupos etarios, se halló que dichos efectos estuvieron concentrados en los varones entre 12 y 19 años (cursando secundaria).

En general, los resultados encontrados en consumo per cápita y acceso a los servicios (con excepción de los servicios de salud) van en línea con lo esperado y la evidencia internacional. Sin embargo, pareciera que los cambios en los mecanismos intermedios no se habrían materializado en cambios sobre los estados nutricionales; entre otras cosas, debido a las limitaciones desde el lado de la oferta.

\section{CASO 3. EVALUACIÓN DE IMPACTO AL PROGRAMA NACIONAL DE ASISTENCIA SOLIDARIA PENSIÓN $65^{(25)}$}

Pensión 65 tiene como objetivo fundamental proporcionar seguridad económica a los adultos mayores de 65 años que viven en pobreza extrema, para asegurar una mejor calidad de vida y bienestar. Para lograrlo, entrega 
Tabla 3. Impactos estimados de JUNTOS^

\begin{tabular}{|c|c|c|c|}
\hline \multirow{2}{*}{ Indicadores de resultado } & \multicolumn{3}{|c|}{ Treatment on treated (TOT) } \\
\hline & $\mathbf{N}$ & Coeficiente & Error estándar \\
\hline \multicolumn{4}{|l|}{ Indicadores de gasto y pobreza } \\
\hline Logaritmo del gasto per cápita & 4154 & $0,06^{*}$ & 0,03 \\
\hline Logaritmo del gasto per cápita en alimentos & 4149 & $0,09^{* *}$ & 0,04 \\
\hline Logaritmo del gasto básico per cápita & 4153 & $0,08^{*}$ & 0,04 \\
\hline Hogar pobre & 4154 & 0,01 & 0,02 \\
\hline Brecha de pobreza & 4154 & $-0,02$ & 0,01 \\
\hline Severidad de la pobreza & 4154 & $-0,03^{* *}$ & 0,01 \\
\hline Hogar pobre extremo & 4154 & $-0,03$ & 0,03 \\
\hline Brecha de la pobreza extrema & 4154 & $-0,03^{* *}$ & 0,01 \\
\hline Severidad de la pobreza extrema & 4154 & $-0,03^{* * *}$ & 0,01 \\
\hline \multicolumn{4}{|l|}{ Indicadores de salud y desnutrición } \\
\hline Parto institucional & 2000 & $-0,01$ & 0,04 \\
\hline Talla al nacer (centímetros) & 1175 & 0,74 & 0,66 \\
\hline Peso al nacer del último hijo (gramos) & 1611 & 8,62 & 64,19 \\
\hline Bajo peso al nacer (último nacido vivo) & 1611 & $-0,04$ & 0,04 \\
\hline Número de controles CRED (menores de 60 meses de edad) & 1405 & 1,58 & 1,39 \\
\hline Cumple adecuadamente las pautas de controles CRED & 1388 & $0,21^{* * *}$ & 0,07 \\
\hline Vacunación menores de 36 meses de edad & 1057 & 0,05 & 0,11 \\
\hline Z-score (talla/edad) & 2557 & 0,02 & 0,08 \\
\hline Desnutrición crónica (z_score<-2DE) & 2557 & 0,03 & 0,03 \\
\hline Desnutrición crónica extrema (z_score<-3DE) & 2557 & 0,00 & 0,02 \\
\hline \multicolumn{4}{|l|}{ Indicadores de educación y distribución del tiempo } \\
\hline Asistencia escolar (personas de 6 a 19 años) & 7303 & $0,05^{\star * *}$ & 0,02 \\
\hline Años de educación (personas de 6 a 19 años) & 7722 & $0,18^{* * *}$ & 0,06 \\
\hline Deserción escolar (personas de 6 a 19 años) & 7303 & $-0,04^{\star * \star}$ & 0,02 \\
\hline Asistencia escolar (personas de 12 a 19 años) & 3016 & $0,08^{\star * \star}$ & 0,03 \\
\hline Años de educación (personas de 12 a 19 años) & 3435 & $0,04^{* * *}$ & 0,01 \\
\hline Deserción escolar (personas de 12 a 19 años) & 3016 & $-0,09^{* \star *}$ & 0,03 \\
\hline Asistencia escolar (mujeres de 12 a 19 años) & 1499 & 0,00 & 0,02 \\
\hline Deserción escolar (mujeres de 12 a 19 años) & 1499 & 0,02 & 0,00 \\
\hline Asistencia escolar (varones de 12 a 19 años) & 1517 & $0,02^{* * *}$ & 0,01 \\
\hline Deserción escolar (varones de 12 a 19 años) & 1517 & $-0,07^{* *}$ & 0,03 \\
\hline Nota promedio desaprobatoria - menos de 11 (personas de 6 a 19 años) & 6440 & 0,01 & 0,01 \\
\hline Distribución del tiempo - tareas cotidianas (personas de 6 a 19 años) & 3302 & $-0,01^{* * *}$ & 0,00 \\
\hline Distribución del tiempo - actividades educativas (personas de 6 a 19 años) & 3302 & $0,01^{\star *}$ & 0,00 \\
\hline
\end{tabular}

*** Valor $p<0,01,{ }^{* *}$ Valor $p<0,05,{ }^{*}$ Valor $p<0,1$

${ }^{\wedge}$ Metodología: diferencias en diferencias con efectos fijos por hogar y variables de control de individuales y de la vivienda

Fuente: elaboración propia a partir de los informes de evaluación 
subvenciones económicas bimensuales equivalentes a S/ 250 a sus usuarios. Adicionalmente, promueve su acceso a servicios públicos a través de las acciones de articulación intersectorial e intergubernamental que realiza (salud, identificación, entre otras).

La evaluación de impacto tuvo como objetivo medir los efectos de Pensión 65 sobre el bienestar de sus usuarios y el de sus respectivos hogares. Para ello, se definieron indicadores vinculados a los objetivos del programa, al modelo causal y la literatura internacional, previamente documentados por otros autores en otros países (16, 26-28). En particular, además del consumo se evaluaron cambios en la oferta laboral, bienestar emocional y salud física del adulto mayor (Tabla 4).

La metodología empleada fue la de un diseño de regresión discontinua basado en el criterio de elegibilidad para el programa: la condición de pobreza. Un adulto mayor es elegible sí y solo si su puntuación de pobreza está por debajo del umbral de elegibilidad definido por el sistema de focalización de hogares (SISFOH), además de otros criterios de elegibilidad definidos por Pensión 65. Por lo tanto, se presume que los adultos mayores cuya situación de pobreza estuvo un poco por debajo del umbral de elegibilidad son similares a los adultos mayores cuya situación de pobreza estuvo un poco por encima de dicho umbral y que la única diferencia entre ambos es que los primeros reciben el tratamiento y los segundos no lo reciben.

La muestra de la evaluación estuvo conformada por 3500 adultos mayores de 65 y de hasta 80 años quienes, a agosto del 2012, cumplían los criterios de elegibilidad del programa y tenían un puntaje de pobreza ubicado en el rango de +/- 0,3 desviaciones estándar alrededor del umbral (rango establecido para asegurar similitud de los grupos de tratamiento y control). Con ello, la evaluación estimó el efecto del tratamiento promedio local comparando los resultados de los adultos mayores potenciales usuarios del programa con las de aquellos que no cumplían la condición de pobreza por ubicarse hasta 0,3 desviaciones estándar por encima del umbral.

El análisis empleó la Encuesta de Salud y Bienestar del Adulto Mayor, diseñada específicamente para la evaluación y aplicada en 12 regiones en los años 2012 y 2015. La Tabla 1 muestra que, en sus mediciones basales, tanto el grupo control como tratamiento tenían valores promedio estadísticamente equivalentes. En particular, se encontró que los adultos mayores trabajaban a pesar de su avanzada edad (en promedio 16 a 17 horas semanales) y presentaban enfermedades crónicas degenerativas, como artrosis / reumatismo y osteoporosis y otras como el colesterol alto, diabetes y enfermedades del sistema nervioso, pero con rangos normales en los indicadores asociados a
Tabla 4. Impactos estimados de Pensión $65^{\wedge}$

\begin{tabular}{|c|c|c|c|}
\hline \multirow[b]{2}{*}{$\begin{array}{l}\text { Indicadores de } \\
\text { resultado }\end{array}$} & \multicolumn{3}{|c|}{ Intention-to-treat (ITT) } \\
\hline & $\mathbf{N}$ & $\beta$ & $\begin{array}{c}\text { Error } \\
\text { estándar }\end{array}$ \\
\hline \multicolumn{4}{|l|}{$\begin{array}{l}\text { Oferta de trabajo } \\
\text { individual }\end{array}$} \\
\hline $\begin{array}{l}\text { Trabajó la última } \\
\text { semana }\end{array}$ & 3342 & $-0,03$ & 0,03 \\
\hline $\begin{array}{l}\text { Horas trabajadas- } \\
\text { trabajó la última semana }\end{array}$ & 3342 & $-1,39^{* *}$ & 0,51 \\
\hline $\begin{array}{l}\text { Trabajó la última } \\
\text { semana por un salario }\end{array}$ & 3342 & $-0,06^{\star * *}$ & 0,02 \\
\hline $\begin{array}{l}\text { Horas trabajadas- } \\
\text { trabajó la última semana } \\
\text { por un salario }\end{array}$ & 3342 & $-1,08$ & 0,76 \\
\hline Ingresos laborales & 3342 & $-5,73^{\star * *}$ & 1,76 \\
\hline \multicolumn{4}{|l|}{ Salud y el bienestar } \\
\hline Hipertensión & 3342 & $-0,07^{*}$ & 0,03 \\
\hline Peso & 3342 & $-0,79$ & 1,38 \\
\hline IMC & 3342 & $-0,06$ & 0,13 \\
\hline Puntaje de la memoria & 3342 & 0,11 & 0,24 \\
\hline Salud física & 3342 & $-0,03$ & 0,06 \\
\hline Depresión & 3342 & $-0,04^{*}$ & 0,02 \\
\hline Satisfacción & 3342 & 0,00 & 0,02 \\
\hline Empoderamiento & 3342 & 0,03 & 0,02 \\
\hline Contribución & 3342 & $0,11^{* \star *}$ & 0,02 \\
\hline Autovaloración & 3342 & $0,04^{* * *}$ & 0,01 \\
\hline Bienestar & 3342 & $0,17^{* * *}$ & 0,03 \\
\hline \multicolumn{4}{|l|}{$\begin{array}{l}\text { Ingresos y gastos del } \\
\text { hogar }\end{array}$} \\
\hline $\begin{array}{l}\text { Ingreso laboral } \\
\text { equivalente por adulto }\end{array}$ & 2584 & 4,99 & 6,73 \\
\hline $\begin{array}{l}\text { Ingreso laboral } \\
\text { equivalente por adulto } \\
\text { excluyendo al adulto } \\
\text { mayor }\end{array}$ & 2584 & 6,16 & 6,46 \\
\hline $\begin{array}{l}\text { Gastos del hogar } \\
\text { equivalente por adulto }\end{array}$ & 2584 & $18,05^{\star * *}$ & 3,94 \\
\hline $\begin{array}{l}\text { Gastos del hogar en } \\
\text { alimentos equivalente } \\
\text { por adulto }\end{array}$ & 2584 & $12,16^{* * *}$ & 3,21 \\
\hline $\begin{array}{l}\text { Gastos del hogar en no } \\
\text { alimentos equivalente } \\
\text { por adulto }\end{array}$ & 2584 & $5,89^{* *}$ & 1,97 \\
\hline
\end{tabular}

*** Valor $p<0,01,{ }^{* *}$ Valor $p<0,05,{ }^{*}$ Valor $p<0,1$

Ho: $=0$

${ }^{\wedge}$ Metodología: regresión discontinua con efectos fijos por conglomerados y variables de control

Fuente: elaboración propia a partir de los informes de evaluación 
riesgo cardiovascular como índice de masa corporal y circunferencia de cintura. Además, entre las razones que estos dieron para justificar la falta de tratamiento de dichas enfermedades, la principal fue la insuficiencia de recursos.

Los resultados hallados muestran que el programa incrementó el gasto per cápita del hogar, cuya mayor proporción se destinó a alimentos. También mejoró la salud emocional del adulto mayor, medido por la reducción en el nivel de depresión y mejoras en la autovaloración y percepción de contribución al hogar (Tabla 4), similar a los efectos obtenidos en México.

Sin embargo, pese a que el programa otorga elegibilidad para atención médica integral gratuita y que se registró un incremento en la proporción de adultos mayores afiliados al seguro de salud, no se reportó un mayor uso de los servicios de salud. En esta línea tampoco se encontraron efectos en el estado de salud física (no se reportaron cambios en hipertensión, IMC, circunferencia de cintura y puntuación de memoria ni sobre la percepción del estado de salud del usuario).

De otro lado, se encontró que la proporción de adultos mayores que trabajaba por necesidad se redujo, asociado a la reducción del porcentaje de participación del ingreso laboral, respecto de los ingresos totales.

\section{DISCUSIÓN}

Los efectos de los programas evaluados en la mejora del acceso a servicios de salud y estado de salud física de su población usuaria no son muy alentadores.

Si bien Cuna Más parece tener potencial para mejorar las dimensiones cognitivas y de comunicación de los niños menores de 3 años, no parece tener efectos sobre el conocimiento de las madres en prácticas de cuidado infantil relacionado a la alimentación y cuidado del menor ni tampoco sobre nivel nutricional de los niños. Cabe señalar que, si bien el nivel nutricional de los niños no era un resultado del programa, es una condición necesaria para mejorar las dimensiones del desarrollo infantil. Por ello, el currículo de las visitas buscó, entre otras cosas, fomentar buenas prácticas de cuidado y salud infantil. En ese sentido, se sugiere que los contenidos técnicos de los mensajes que se quieran fomentar (salud, nutrición e higiene) sean fortalecidos, desarrollados y coordinados con el MINSA.

Por su parte, Juntos, que atiende a gestantes, niños adolescentes y jóvenes hasta 19 años de edad sí tiene como resultado específico el acceso de su población usuaria a los servicios de salud y educación. Sin embargo, el programa no tuvo efectos significativos en la mayoría de indicadores vinculados a acceso a servicios de salud prenatal ni en el estado de salud materno infantil. Al parecer, las mejoras encontradas en consumo, consumo de alimentos y gasto per cápita y asistencia a controles de crecimiento no se habrían llegado a materializar en cambios sobre los estados nutricionales. Estos resultados advierten sobre la pertinencia de las condicionalidades vinculadas al acceso a salud, su proceso de verificación y la calidad de la oferta de los servicios.

Finalmente, aunque el objetivo del programa Pensión 65 se centra en la mejora de la seguridad económica del adulto mayor, los resultados del estudio muestran que, además del incremento en el consumo del hogar, se logró mejorar el bienestar emocional del adulto mayor (tasa de depresión, satisfacción personal y empoderamiento del adulto mayor). Sin embargo, la ausencia de impactos en el uso de servicios de salud y en la salud física del adulto mayor alertan sobre la necesidad de fortalecer las acciones de articulación intersectorial e intergubernamental para la entrega de servicios complementarios a la subvención económica, especialmente en las estrategias de promoción de la salud y atención primaria, con enfoque gerontológico, y de género pues la subvención económica cubriría necesidades de consumo familiar pero no de acceso a salud.

Contribución de los autores: VC, GC y DC, han participado en la concepción del artículo, recolección de resultados y redacción del artículo. VC además participó en la aprobación de la versión final y revisión crítica del artículo.

Agradecimiento: a Juan José Cueto por su valiosa colaboración en la elaboración del artículo.

Conflictos de interés: no existen conflictos de interés en la publicación de este artículo.

Fuentes de financiamiento: autofinanciado.

\section{REFERENCIAS BIBLIOGRÁFICAS}

1. Attanasio O, Fernández C, Fitzsimons E, Grantham-McGregor S, Meghir C, Rubio-Codina M. Using the infrastructure of a conditional cash transfer program to deliver a scalable integrated early child development program in $\mathrm{Co}$ - lombia: cluster randomized controlled trial. The BMJ. 2014; 1-12.

2. Berhman J, Cheng Y, Todd P. Evaluating preschool programs when length of exposure to the Program Varies: A nonparametric approach. Review of Economics and Statistics. 2004; 86(1): 108-132.

3. Bernal R, Fernandez, C, Flórez, C, Gaviria, A, Ocampo, P, Samper, B, Sánchez, F. Evaluación de impacto del Programa Hogares Comunitarios de Bienestar del ICBF. [Internet]. Bogotá, 
Colombia: Centro de Estudios sobre Desarrollo Económico; 2009 [citado en junio de 2015] Disponible en: https://economia.uniandes.edu.co/ components/com_booklibrary/ebooks/ dcede2009-16.pdf

4. Bastagli F, Hagen-Zanker J, Harman, L, Sturge, G, Braca, V, Schmidt, Pellerano, L. Cash transfers: what does the evidence say? A rigorous review of impacts and the role of design and implementation features. [Internet]. 2016 [citado en julio de 2016] Disponible en: https:// www.odi.org/publications/10505cash-transfers-what-does-evidence-sayrigorous-review-impacts-and-role-designand-implementation

5. Fizbein, A. y N. Schady. Conditional Cash Transfers: Reducing Present and Future Poverty. [Internet]. Washington DC, United States: World Bank; 2009 [citado en abril de 2016] Disponible en: http:// siteresources.worldbank.org/INTCCT/ Resources/5757608-1234228266004/ PRR-CCT_web_noembargo.pdf

6. Veras, F, Perez, R, Issamu, G. Achievements and Shortfalls of Conditional Cash Transfers: Impact Evaluation of Paraguay's Tekoporã Programme. [Internet]. 2008 [citado en abril de 2016] Disponible en: http://www.ipc-undp.org/pub/IPCEvaluationNote3.pdf

7. Gertler P, Patrinos, H, Rubio-Codina, M. [Internet]. Washington DC, United States: World Bank; 2006 [citado en junio de 2016] Disponible en: http://siteresources.worldbank.org/ INTLACREGTOPEDUCATION/ Resources/Conafe_ Oportunidades_ AAA.pdf

8. DPN. Programa Familias en Acción Impactos en capital humano y evaluación costo beneficio del programa. [Internet]. Bogotá, Colombia: DPN; 2008 [Citado en junio de 2015] Disponible en https:// colaboracion.dnp.gov.co/CDT/Prensa/ Publicaciones/Evaluacion-politicaspublicas-6.pdf

9. Gertler P. Do Conditional Cash Transfers Improve Child Health? Evidence from PROGRESA's Control Randomized Experiment. The American Economic Review. 2004; 94(2): 336-341

10. Berhman J, Hoddinott J. Programme Evaluation with Unobserved Heterogeneity and Selective Implementation: The
Mexican PROGRESA Impact on Child Nutrition. Oxford Bulletin of Economics and Statistics. 2005; 67(4): 547-569.

11. Paxson C, Schady N. Does Money Matter? The Effects of Cash Transfer son Child Health and Development in Rural Ecuador. Economic Development and Cultural Change. 2010; 59(1): 187-229.

12. Ponce J, Bedi A. The impact of a Cash Transfer Program on Cogntive Achievment: The Bono de Desarrollo Humano of Ecuador. Economics of Education Review. 2010; 29(1): 116-125.

13. Evangelista I, Filho C. Old-age Benefits and Retirement Decisions of Rural Elderly in Brazil. Journal of Development Economics. 2008; 86: 129-146.

14. Bosch M, Guajardo, J. Labor Market Impacts of Non-Contributory Pensions: The Case of Argentina's Moratorium. IDB [Internet]. Washington DC, United States of America: IDB; 2012 [citado en junio de 2016] Disponible en https://publications. iadb.org/handle/11319/4094

15. Bosch M, Melguizo, A, Pagés, C. Mejores pensiones mejores trabajos: Hacia la cobertura universal en América Latina y el Caribe. IDB [Internet]. Washington DC, United States of America: IDB; 2013 [Citado en junio de 2005] Disponible en: https://publications.iadb.org/handle/11319/462

16. Galiani S, Gertler P, Bando R. Noncontributory pensions. Labour Economics. 2016; 38: 47-58.

17. MIDIS [Internet]. Lima, Perú: MIDIS; 2012 [Citado en junio de 2005] Disponible en: http://www.midis.gob.pe/files/ estrategianacionaldedesarrolloeinclusinsocialincluirparacrecer.pdf

18. BID, MEF y MIDIS. Informe de línea de base para la evaluación de impacto del PNCM.2014. Mimeo.

19. BID y MEF. Resultados de la evaluación de impacto del Servicio de Acompañamiento a Familias del Programa Nacional Cuna Más. 2016. Mimeo.

20. Troude P, Squires J, Helias LF, J Bouyer, DeLa Rechebrochard, E. Ages and stages questionnaires: Feasibility of postal surveys for child follow-up. Early Human Development. 2011; 87(10):671-676.
21. Monge A, Campana Y, Sainfield J. Servicio de Consultoría para el Análisis de Resultados para Estimar los Efectos Directamente Atribuibles del Programa Nacional de Apoyo Directo a los Más Pobres JUNTOS. 2017. Mimeo.

22. Perova E, Vakis R. Welfare impacts of the "Juntos" Program in Peru: Evidence from a non-experimental evaluation. [Internet]. Washington DC, World Bank; 2009 [citado en febrero de 2016]. Disponible en: http://www.juntos. gob.pe/modulos/mod_legal/archivos/ Evaluacion_Cuasi-Experimental1.pdf

23. Sánchez A, Jaramillo M. Impacto del programa Juntos sobre la nutrición temprana. Revista Estudios Económicos. 2012; 23: 53-66.

24. Vargas P. Wealthier and Healthier: Redesigning the Peruvian Conditional Cash Transfer Program "JUNTOS" to achieve better health outcomes on infants [Tesis de maestría]. Cambridge: Harvard Kennedy School of Government; 2013.

25. Gertler P, Galiani, S. "Evaluación de Impacto del Programa Pensión 65: Nueva evidencia causal de las pensiones no contributivas en Perú". 2016. Mimeo

26. Duflo, E. "Grandmothers and Granddaughters: Old-Age Pensions and Intrahousehold Allocation in South Africa”. World Bank [Internet]. Washington DC, United States: World Bank; 2003 [Citado en mayo de 2015] Disponible en: https://openknowledge.worldbank.org/bitstream/ handle/10986/17173/774010JRN020030PUBLIC00Grandmothers. pdf ? sequence $=1 \&$ is Allowed $=y$

27. Hamoudi A, Thomas D. Endogenous coresidence and program incidence: South Africa's Old Age Pension. Journal of Development Economics. 2014; 109: 30-37.

28. Fan E. Who Benefits from Public Old Age Pensions? Evidence from Targeted Program. Economic Development and Cultural Change. 2010; 58 (2): 297-322.

Correspondencia: Viviana Cruzado de la Vega Direccción: Calle Berlin 974 dpto 701, Miraflores, Lima, Perú.

Teléfono: 986642272

Correo electrónico: cruzado.viviana@gmail.com 